\section{Explosão da mortalidade no epicentro amazônico da epidemia de COVID-19}

\section{Explosion in mortality in the Amazonian epicenter of the COVID-19 epidemic}

\section{Explosión de la mortalidad en el epicentro amazónico de la epidemia de COVID-19}

COMUNICAÇÃO BREVE

BRIEF COMMUNICATION

\section{Resumo}

Manaus, capital do estado brasileiro do Amazonas, é o atual epicentro da epidemia na Amazônia com um aumento repentino de mortes que preocupa gestores e sociedade. O objetivo do estudo foi analisar o excesso na mortalidade geral, segundo Semanas Epidemiológicas (SE), visando a identificar mudanças potencialmente associadas à epidemia em Manaus. Dados de mortalidade geral e grupos de causas foram obtidos na Central de Informações do Registro Civil Nacional e no Sistema de Informações sobre Mortalidade, para 2018, 2019 e 2020. Analisou-se faixa etária, sexo, local de ocorrência do óbito, SE, ano-calendário e causas de morte. Calcularam-se razões entre as mortes ocorridas em 2019/2018 e 2020/2019 para avaliar o excesso de mortes, com intervalos de confiança no nível de 5\%. Não observou-se excesso de mortalidade geral significativo nas razões 2019/2018, independentemente da SE. Já as razões de 2020/2019 passaram de 1,0 (IC95\%: 0,9-1,3) na SE 12 para 4,6 (IC95\%: 3,9-5,3) na SE 17. Observou-se excesso de mortalidade geral com a progressão da idade, especialmente em indivíduos com 60 anos e mais, os quais concentraram 69,1\% (IC95\%: 66,8-71,4) das mortes. A razão 2020/2019 para óbitos em domicílio/via pública foi de 1,1 (IC95\%: 0,7-1,8) na SE 12 e de 7,8 (IC95\%: 5,4-11,2) na SE 17. A explosão da mortalidade geral em Manaus e a elevada proporção de óbitos em domicílio/via pública expõe a gravidade da epidemia em contextos de grande desigualdade social e fraca efetividade de ações governamentais, em especial aquelas voltadas ao enfrentamento das desigualdades sociais e para a garantia e fortalecimento do Sistema Único de Saúde.

COVID-19; Populações Vulneráveis; Vigilância em Saúde Pública; Mortalidade
Jesem Douglas Yamall Orellana 1

Geraldo Marcelo da Cunha 2

Lihsieh Marrero 3

Bernardo Lessa Horta 4

Iuri da Costa Leite 2

doi: 10.1590/0102-311X00120020
Correspondência

J.D. Y. Orellana

Instituto Leônidas e Maria Deane, Fundação Oswaldo Cruz.

Rua Teresina 476, 2o andar, sala 203, Manaus, AM

69057-070, Brasil.

jesem.orellana@fiocruz.br

1 Instituto Leônidas e Maria Deane, Fundação Oswaldo Cruz, Manaus, Brasil.

2 Escola Nacional de Saúde Pública Sergio Arouca, Fundação Oswaldo Cruz, Rio de Janeiro, Brasil.

3 Universidade do Estado do Amazonas, Manaus, Brasil.

${ }_{4}^{4}$ Centro de Pesquisas Epidemiológicas, Universidade Federal de Pelotas, Pelotas, Brasil. 


\section{Introdução}

Em 20 de maio de 2020, dois meses após a Organização Mundial da Saúde (OMS) declarar a COVID-19 como pandemia, em torno de cinco milhões de casos e aproximadamente 320 mil óbitos foram registrados em 216 países/áreas/territórios. Atualmente, mesmo havendo fortes evidências de subestimação de suas estatísticas oficiais 1, o Brasil é o terceiro país mais afetado, com cerca de 280 mil casos e 18 mil mortes (World Health Organization. WHO coronavirus disease (COVID-19) dashboard. https://covid19.who.int/, acessado em 20/Mai/2020).

A distribuição dos óbitos por COVID-19 reflete a heterogeneidade geográfica e social do país, com apenas cinco estados respondendo por $81 \%$ das ocorrências: São Paulo, Rio de Janeiro, Ceará, Pernambuco e Amazonas, sendo que este último apresenta a maior proporção de infectados, 10,6\% (IC95\%: 8,8\%-12,1\%) 2 .

No caso do Estado do Amazonas, situado na Amazônia brasileira, região que ocupa aproximadamente $60 \%$ do território do nacional, cuja população tem sido historicamente submetida a condições de pobreza e desigualdade social 3. Um estudo sobre a Região Metropolitana de Manaus constatou elevada desigualdade no acesso aos serviços de saúde 4 . Essa é uma realidade comum para populações vivendo em regiões de acesso remoto e terras indígenas 5 , cuja vulnerabilidade social e econômica restringe sua mobilidade espacial no território, tornando-as mais susceptíveis à dramática disseminação da COVID-19, em especial de suas formas graves.

Há mais de quatro semanas, Manaus apresenta sinais de esgotamento na rede pública hospitalar, devido ao rápido aumento do número de casos de COVID-19. Só na primeira quinzena de maio, foram quase sete mil novos casos, o dobro do número até então identificado. Ademais, de 19 a 28 de abril, a média diária de sepultamentos foi de 123, valor quatro vezes maior do que a média diária de 2019 6. Chama atenção que a média diária de mortes por COVID-19, reconhecida pelos serviços de saúde no mesmo período, tenha sido de apenas 14 óbitos, sugerindo ampla subnotificação, um problema mundialmente reconhecido, sobretudo em regiões de precária testagem e serviços de saúde deficitários 7,8 .

Apesar das incertezas sobre a mortalidade específica por COVID-19 9, indicadores do excesso de óbitos constituem parâmetros mais objetivos e comparáveis para avaliar o impacto da epidemia sobre a mortalidade 10. Portanto, o objetivo deste estudo foi analisar o excesso na mortalidade geral, segundo Semanas Epidemiológicas (SE), visando a identificar mudanças no risco de morte, potencialmente associadas à epidemia.

\section{Métodos}

\section{Tipo de estudo e fontes de dados}

Estudo transversal com dados de mortalidade oriundos da Central de Informações do Registro Civil (CRC) Nacional (https://sistema.registrocivil.org.br) e do Sistema de Informações sobre Mortalidade (SIM. http://www2.datasus.gov.br).

Devido ao cenário pandêmico, a CRC Nacional estruturou o Painel Registral COVID-19 (Portal da Transparência. https://transparencia.registrocivil.org.br/registral-COVID, acessado em 10/Mai/2020), cujo objetivo é fornecer dados sobre as causas de mortes de Declarações de Óbitos (DO) lavradas em Cartórios, as quais representam a totalidade de mortes naturais, aqui tratadas como proxy de mortalidade geral. A atualização dos dados é diária e obedece a prazos legais. O tempo entre o registro do óbito e o seu envio à plataforma do Painel Registral é de até 14 dias, quando o dado passa a ser público.

\section{Definições operacionais}

De acordo com os critérios da CRC Nacional, uma morte por COVID-19, suspeita ou confirmada, corresponde aos óbitos em que a DO fez menção aos termos COVID-19, "coronavírus" ou "novo coronavírus” nas partes I (linhas a, b, c, d) ou II (outras condições mórbidas preexistentes e sem relação direta com a morte, que não entraram na sequência causal declarada na parte I). Além da COVID-19, 
outras possíveis causas foram consideradas, de acordo com a CRC: síndrome respiratória aguda grave (SRAG); pneumonia; septicemia; e insuficiência respiratória. Óbitos não classificados em nenhuma das condições anteriores são incluídos na categoria "demais causas". Por fim, as mortes "indeterminadas" (causas de mortes ligadas a doenças respiratórias, mas não conclusivas) representaram menos de $1 \%$ da amostra avaliada e não foram apresentadas separadamente.

Os dados da CRC Nacional foram atualizados em 19 de maio de 2020, 66 dias após o início da SE 12 e 24 dias depois do último dia da SE 17. O início da SE 12 remete aos dois dias subsequentes da confirmação do primeiro caso de COVID-19 e aos 15 dias anteriores à ocorrência da primeira morte pela doença em Manaus.

Para fins de comparação, também foram utilizados dados sobre mortalidade geral do SIM, disponibilizados para as SE consideradas, em 2018, na cidade de Manaus.

\section{Variáveis incluídas}

Faixa etária, sexo, ocorrência do óbito no domicílio ou via pública, SE, ano-calendário e causas de morte. As mortes por SRAG, pneumonia (PNM) e insuficiência respiratória (IR) foram agregadas em uma única variável denominada "SRAG+PNM+IR", a qual exclui o grupo de mortes suspeitas ou confirmadas por COVID-19 e de acordo com os critérios da CRC Nacional.

\section{Análise de dados}

Foram calculadas as razões entre o total de mortes ocorridas em 2019 e 2018, 2019/2018, e em 2020 e 2019, 2020/2019, nas SE de 12 a 17, em Manaus. Também foram calculadas as razões de mortes 2020/2019 nas SE de 14 a 17, estratificadas por sexo, faixa etária e causa de mortalidade. Para as razões de mortalidade foram calculados intervalos de confiança, com nível de significância de 5\%. As análises foram efetuadas no software R, versão 3.6.1 (http://www.r-project.org).

\section{Resultados}

Em 2018, segundo o SIM, o número médio de mortes semanal, em Manaus foi de 230, valor próximo do observado nos primeiros 70 dias de 2019 e 2020, segundo informações da CRC nacional, respectivamente, 225 e 218 , óbitos.

Conforme pode ser visto na Figura 1, a razão de mortalidade geral, 2019/2018, é muito próxima a 1, independentemente da SE; já a razão 2020/2019 apresenta um comportamento semelhante apenas entre as SE 12 e 14, com aumento expressivo nas semanas subsequentes. De forma que a razão na SE 12 passa de 1,0 (IC95\%: 0,9-1,3) para 4,6 (IC95\%: 3,9-5,3) na SE 17.

As razões de mortalidade geral, 2020/2019, segundo faixa etária, não foram estatisticamente significativas em menores de 40 anos do sexo masculino, bem como nos menores de 30 anos do sexo feminino e em ambos os sexos, expondo, a partir de então, o excesso de mortalidade em 2020, principalmente no sexo masculino (Tabela 1).

Quando comparamos a razão de mortalidade 2020/2019 segundo grupos de causas de morte, observamos um gradativo aumento ao longo das semanas, especialmente a partir da SE 15, para as mortes por SRAG+PNM+IR (Figura 2). No grupo das “demais causas”, a razão de mortalidade mantém-se próxima de 1 até a SE 14, e então aumenta, atingindo o patamar de 3 na SE 17.

No que diz respeito à distribuição dos óbitos entre os grupos etários, 69,1\% deles ocorreram entre os indivíduos com 60 anos ou mais (IC95\%: 66,8-71,4). A razão de óbitos em domicílio/via pública (43/38) na SE 12 foi de 1,1 (IC95\%: 0,7-1,8). Essa mesma razão (268/33) foi de 8,1 (IC95\%: 5,7-11,7) na SE 17 (dados não apresentados). 
Figura 1

Razões de mortalidade geral, para os anos de 2019/2018 e 2020/2019, de acordo com Semanas Epidemiológicas (SE). Manaus, Amazonas, Brasil.

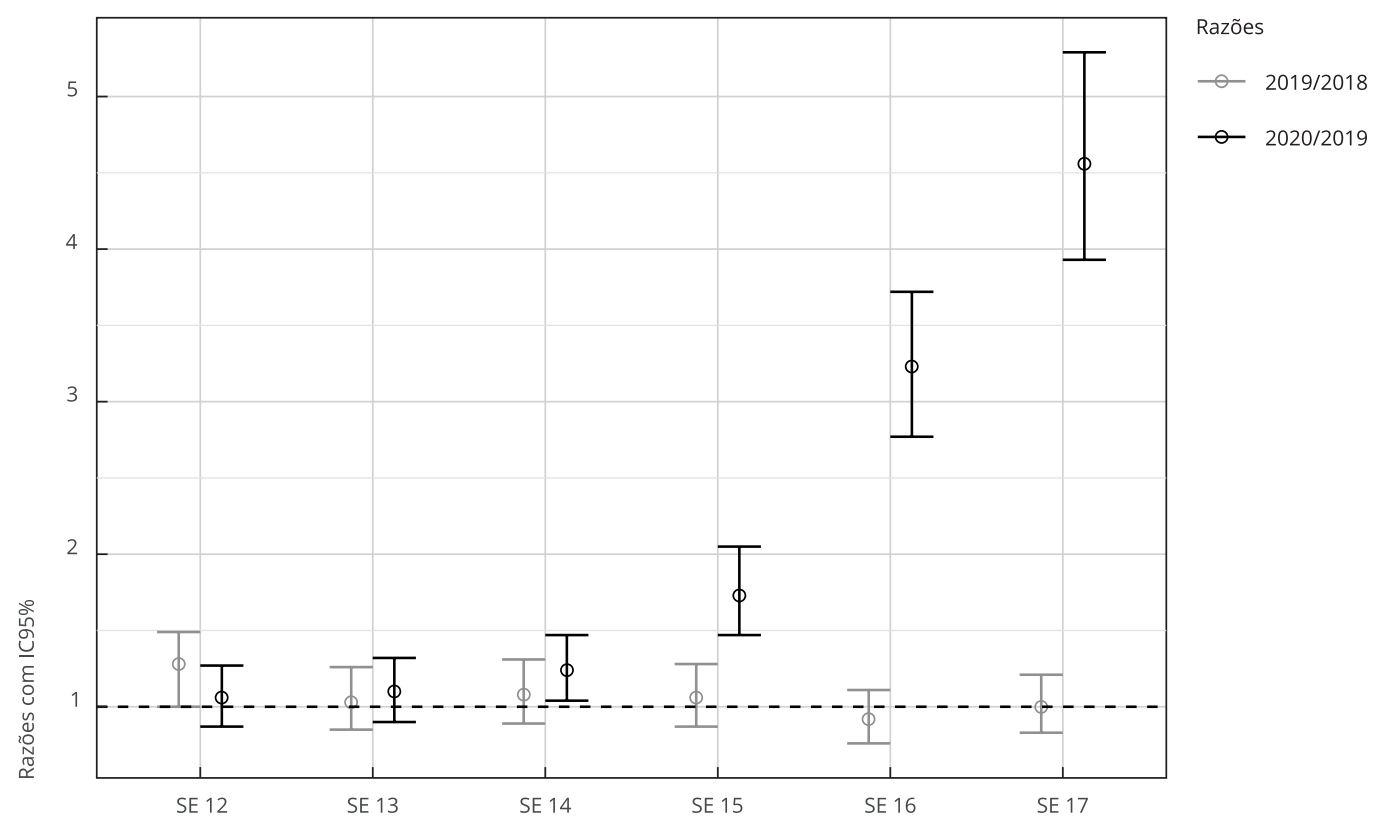

Fontes: Central de Informações do Registro Civil (CRC) Nacional (https://sistema.registrocivil.org.br) e Sistema de Informações sobre Mortalidade (SIM. http://www2.datasus.gov.br).

Tabela 1

Frequências de mortalidade geral e suas respectivas razões e intervalos de confiança (IC95\%), referentes às Semanas Epidemiológicas (SE) 14 a 17, segundo faixa etária e sexo. Manaus, Amazonas, Brasil.

\begin{tabular}{lcccc}
\hline Sexo/Faixa etária (anos) & $\mathbf{2 0 2 0}(\mathbf{n})$ & $\mathbf{2 0 1 9}(\mathbf{n})$ & Razão & IC95\% \\
\hline Masculino & & & & \\
$0-9$ & 51 & 61 & 0,84 & $0,58-1,21$ \\
$10-19$ & 9 & 6 & 1,50 & $0,53-4,21$ \\
$20-29$ & 35 & 27 & 1,30 & $0,78-2,14$ \\
$30-39$ & 54 & 37 & 1,46 & $0,96-2,22$ \\
$40-49$ & 110 & 38 & 2,89 & $2,00-4,19$ \\
$50-59$ & 195 & 42 & 4,64 & $3,33-6,48$ \\
$60-69$ & 315 & 87 & 3,62 & $2,86-4,59$ \\
$70-79$ & 332 & 91 & 3,65 & $2,89-4,60$ \\
80 e mais & 317 & 89 & 3,56 & $2,82-4,50$ \\
Total & 1.418 & 478 & 2,97 & $2,67-3,29$ \\
\hline
\end{tabular}

(continua) 
Tabela 1 (continuação)

\begin{tabular}{lcccc}
\hline Sexo/Faixa etária (anos) & $\mathbf{2 0 2 0}(\mathbf{n})$ & $\mathbf{2 0 1 9}(\mathbf{n})$ & Razão & IC95\% \\
\hline Feminino & & & & \\
$0-9$ & 48 & 48 & 1,00 & $0,67-1,49$ \\
$10-19$ & 3 & 8 & 0,38 & $0,10-1,41$ \\
$20-29$ & 15 & 11 & 1,36 & $0,63-2,97$ \\
$30-39$ & 30 & 12 & 2,50 & $1,28-4,89$ \\
$40-49$ & 65 & 28 & 2,32 & $1,50-3,62$ \\
$50-59$ & 94 & 38 & 2,47 & $1,69-3,61$ \\
$60-69$ & 175 & 47 & 3,72 & $2,70-5,14$ \\
$70-79$ & 185 & 79 & 2,34 & $1,80-3,05$ \\
80 e mais & 263 & 108 & 2,44 & $1,95-3,04$ \\
Total & 878 & 379 & 2,32 & $2,05-2,61$ \\
Ambos os sexos & & & & \\
$0-9$ & 99 & 109 & 0,91 & $0,69-1,19$ \\
$10-19$ & 12 & 14 & 0,86 & $0,40-1,85$ \\
$20-29$ & 50 & 38 & 1,32 & $0,86-2,01$ \\
$30-39$ & 84 & 49 & 1,71 & $1,21-2,43$ \\
$40-49$ & 175 & 66 & 2,65 & $1,99-3,50$ \\
$50-59$ & 289 & 80 & 3,61 & $2,82-4,63$ \\
$60-69$ & 490 & 134 & 3,66 & $3,02-4,43$ \\
$70-79$ & 517 & 390 & $2,56-3,62$ \\
80 e mais & 580 & 2,04 & $2,50-3,46$ \\
Total & 2.296 & 2,68 & $2,48-2,90$ \\
\hline
\end{tabular}

Fonte: Central de Informações do Registro Civil (CRC) Nacional (https://sistema.registrocivil.org.br).

\section{Figura 2}

Razões de mortalidade por grupos de causas, para os anos de 2020/2019, de acordo com Semanas Epidemiológicas (SE). Manaus, Amazonas, Brasil.

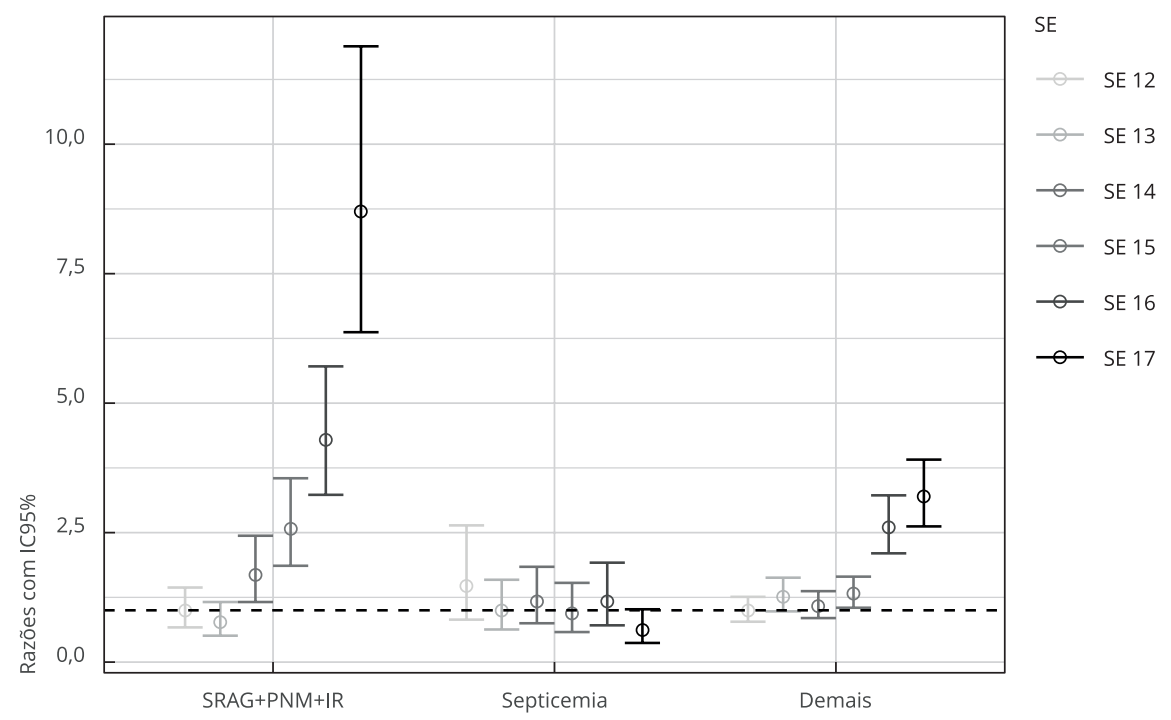

SRAG+PNM+IR: síndrome respiratória agruda grave + pneumonia + insuficiência respiratória.

Fontes: Central de Informações do Registro Civil (CRC) Nacional (https://sistema.registrocivil.org.br) e Sistema de Informações sobre Mortalidade (SIM. http://www2.datasus.gov.br). 


\section{Discussão}

Nossos resultados apontam que o total de óbitos registrados em 2019 é de magnitude similar ao registrado em 2018 entre as SE 12 e 17. Porém, a comparação entre o total de óbitos de 2020 e 2019 revela um excesso de mortalidade a partir da 15a SE de 2020, com esta razão explodindo na SE 17, na qual o número de óbitos é 200\% maior que o observado em 2019.

O aumento de mortes, a partir da SE 15, ocorreu aproximadamente 15 dias após a confirmação dos 30 primeiros casos de COVID-19 em Manaus. Já na SE 17, o número anômalo de mortes coincide com o colapso da rede pública hospitalar. Nesse período, o número médio de sepultamentos diários triplicou. Mortes em casa/via pública também aumentaram, bem como os casos de COVID-19 em municípios vizinhos. Esse conjunto de acontecimentos resulta, provavelmente, de uma grande aceleração da epidemia em Manaus 11, nas semanas anteriores.

A fragilidade da rede de atenção à saúde em Manaus e em municípios vizinhos 4, somada à marcante desigualdade social 3 , ajudam a entender a crítica situação da epidemia de COVID-19. O excesso de mortalidade não está restrito aos países de baixa e média rendas, tendo sido também observado em Nova York, Estados Unidos, e nas províncias de Bérgamo e Bréscia, no norte da Itália 9,12,13.

Em relação à idade, quase $70 \%$ das mortes ocorreram em pessoas com 60 anos ou mais, em consonância com estudos realizados em outros países 9,12,13,14. Nesse segmento populacional, as comorbidades são mais prevalentes e têm sido associadas a um prognóstico pior em casos de internação por COVID-19 15 .

Outro aspecto importante diz respeito aos diferenciais por sexo, com risco de mortalidade maior entre os homens, corroborando achados de outros estudos 16,17. Embora a menor letalidade possa estar associada com a maior percepção dos sintomas da doença e procura por serviços de saúde em mulheres, pois os homens só o fariam nas fases mais graves, em que os recursos terapêuticos geralmente são menores, Zeng et al. 18 argumentam que os maiores níveis de anticorpos IgG em mulheres poderiam explicar, parcialmente, a maior letalidade entre os homens.

Observou-se um aumento explosivo na mortalidade por problemas respiratórios (SRAG+ PNM+IR), complicações comuns da COVID-19 14, durante a epidemia. Constatou-se, também, um aumento significativo na mortalidade pelas demais causas, possível consequência de fatores como: adiamento por parte do paciente de tratamento como meio de evitar exposição ao vírus em hospitais, priorização pelos serviços de saúde ao atendimento de pacientes com COVID-19 19.

Este trabalho tem como ponto forte o uso do indicador de mortalidade geral para estimar o excesso de mortes, o qual parece ser um recurso útil para avaliações rápidas e de baixo custo, além de ser um indicador mais robusto e comparável em cenário pandêmico 10 , pois ao contrário da mortalidade específica por COVID-19, independe de estratégias de testagem adotadas, da organização e do financiamento dos sistemas de saúde, da estrutura demográfica ou do denominador empregado, o que pode fazer com que as estimativas de letalidade variem amplamente 9 . No entanto, a interpretação dos resultados deste estudo deve levar em conta algumas limitações como a ausência de padronização/ revisão das causas de morte nas DO e um possível sub-registro de óbitos na plataforma digital da CRC Nacional, especialmente em 2019, o que poderia superestimar as razões entre o total de óbitos de 2020 e 2019, por exemplo. No entanto, a julgar pelas comparações entre o total de óbitos de 2019 e 2018 , sempre próximas a 1, é possível que essa distorção seja pequena.

A estratégia de análise adotada neste estudo revela de forma inequívoca o elevado excesso da mortalidade em Manaus, expondo a gravidade da epidemia em contextos de grande desigualdade social, fraca efetividade de políticas públicas e fragilidade dos serviços de saúde. Nesse cenário, reforços devem ser envidados rapidamente por gestores das três esferas de governo, de modo a conter ou minorar o efeito deletério da COVID-19, sobretudo em áreas mais precárias, onde o impacto da pandemia sobre a mortalidade tende a ser mais acentuado. 


\section{Colaboradores}

J. D. Y. Orellana, G. M. Cunha e I. C. Leite participaram da concepção, interpretação e redação final do manuscrito. L. Marrero e B. L. Horta participaram da interpretação e revisão crítica do manuscrito.

\section{Informações adicionais}

ORCID: Jesem Douglas Yamall Orellana (00000002-5607-2615); Geraldo Marcelo da Cunha (0000-0001-7128-933X); Lihsieh Marrero (00000002-2856-5682); Bernardo Lessa Horta (00000001-9843-412X); Iuri da Costa Leite (0000-00029136-8948).

\section{Referências}

1. The Lancet. COVID-19 in Brazil: "so what?" Lancet 2020; 395:1461.

2. Mellan TA, Hoeltgebaum HH, Mishra S, Whittaker C, Schnekenberg RP, Gandy A, et al. Estimating COVID-19 cases and reproduction number in Brazil. London: Imperial College London; 2020. (Report 21).

3. Waisbich LT, Shankland A, Bloom G, Coelho VSP. Introduction. The accountability politics of reducing health inequalities: learning from Brazil and Mozambique. Novos Estudos CEBRAP 2019; 38:271-89.

4. GalvãoTF, Tiguman GMB, Caicedo RM, Silva MT. Inequity in utilizing health services in the Brazilian Amazon: a population-based survey, 2015. Int J Health Plann Manage 2019; 34:e1846-53.

5. Ferrante L, Fearnside PM. Protect indigenous peoples from COVID-19. Science 2020; 368:251.

6. Prefeitura de Manaus. Manaus registra quase 2,5 mil sepultamentos em abril. http://www. manaus.am.gov.br/noticia/manaus-registraquase-25-mil-sepultamentos-em-abril/ (acessado em 10/Mai/2020).

7. Ji Y, Ma Z, Peppelenbosch MP, Pan Q. Potential association between COVID-19 mortality and health-care resource availability. Lancet Glob Health 2020; 8:e480.

8. Castro MC, Carvalho LR, Chin T, Kahn R, Franca GV, Macario EM, et al. Demand for hospitalization services for COVID-19 patients in Brazil. medRxiv 2020; 1 abr. https:// www.medrxiv.org/content/10.1101/2020.03. 30.20047662v1.

9. Onder G, Rezza G, Brusaferro S. Case-fatality rate and characteristics of patients dying in relation to COVID-19 in Italy. JAMA 2020; [Online ahead of print].

10. Leon DA, Shkolnikov VM, Smeeth L, Magnus P, Pechholdová M, Jarvis CI. COVID-19: a need for real-time monitoring of weekly excess deaths. Lancet 2020; 395:e81.

11. Programa de Pós-graduação em Ciências do Ambiente e Sustentabilidade na Amazônia, Universidade Federal do Amazonas. Atlas dos objetivos de desenvolvimento sustentável no Amazonas. (Especial COVID-19, 2). https:// edoc.ufam.edu.br/handle/123456789/3198/ (acessado em 03/Mai/2020).
12. Weinberger D, Cohen T, Crawford F, Mostashari F, Olson D, Pitzer VE, et al. Estimating the early death toll of COVID-19 in the United States. medRxiv 2020; 29 abr. https://www. medrxiv.org/content/10.1101/2020.04.15.200 $66431 \mathrm{v} 2$.

13. Ghislandi S, Muttarak R, Sauerberg M, Scotti B. News from the front: excess mortality and life expectancy in two major epicentres of the COVID-19 pandemic in Italy. medRxiv 2020; 13 mai. https://www.medrxiv.org/content/10 $.1101 / 2020.04 .29 .20084335 \mathrm{v} 2$.

14. Shi S, Qin M, Shen B, Cai Y, Liu T, Yang F, et al. Association of cardiac injury with mortality in hospitalized patients with COVID-19 in Wuhan, China. JAMA Cardiol 2020; e200950.

15. Dietz W, Santos-Burgoa C. Obesity and its implications for COVID-19 mortality. Obesity (Silver Spring) 2020; 28:1005.

16. Ciminelli G, Garcia-Mandicó S. COVID-19 in Italy: an analysis of death registry data. VOX 2020; 22 abr. https://voxeu.org/article/ covid-19-italy-analysis-death-registry-data.

17. Huang C, Wang Y, Li X, Ren L, Zhao J, Hu Y, et al. Clinical features of patients infected with 2019 novel coronavirus in Wuhan, China. Lancet 2020; 395:497-506.

18. Zeng F, Dai C, Cai P, Wang J, Xu L, Li J, et al. A comparison study of SARS-COV-2 IgG antibody between male and female COVID-19 patients: a possible reason underlying difference outcome between gender. medRxiv 2020; 27 mar. https://www.medrxiv.org/content/10.11 01/2020.03.26.20040709v1.

19. Vandoros S. Excess mortality during the COVID-19 pandemic: early evidence from England and Wales. medRxiv 2020; 17 mai. https:// www.medrxiv.org/content/10.1101/2020.04. 14.20065706v6. 


\section{Abstract}

Manaus, the capital of the Brazilian State of Amazonas, is the current epicenter of the COVID-19 epidemic in Amazonia. The sharp increase in deaths is a huge concern for health system administrators and society. The study aimed to analyze excess overall mortality according to Epidemiological Week $(E W)$ in order to identify changes potentially associated with the epidemic in Manaus. Overall and cause-specific mortality data were obtained from the Central Database of the National Civil Registry and the Mortality Information System for 2018, 2019, and 2020. The study analyzed age bracket, sex, place of death, EW, calendar year, and causes of death. Ratios were calculated between deaths in 2019/2018 and 2020/2019 to estimate excess deaths, with 5\% confidence intervals. No significant excess overall mortality was seen in the ratios for 2019/2018, independently of EW. Meanwhile, the ratios for 2020/2019 increased from 1.0 (95\%CI: 0.9-1.3) in EW 12 to 4.6 (95\%CI: 3.9-5.3) in EW 17. Excess overall mortality was observed with increasing age, especially in individuals 60 years or older, who accounted for 69.1\% (95\% CI: 66.8-71.4) of the deaths. The ratios for 2020/2019 for deaths at home or on public byways were 1.1 (95\%CI: 0.7-1.8) in EW 12 and 7.8 (95\%CI: 5.4-11.2) in EW 17. The explosion in overall mortality in Manaus and the high proportion of deaths at home or on public byways reveals the epidemic's severity in contexts of heavy social inequality and weak effectiveness of government policies, especially policies meant to deal with social inequalities and strengthen the Unified Health System.

COVID-19; Vulnerable Populations; Public Health Surveillance; Mortality

\section{Resumen}

Manaus, capital del estado brasileño del Amazonas, es el actual epicentro de la epidemia en Amazonia y el aumento repentino de muertes preocupa a gestores y a la sociedad. El objetivo del estudio fue analizar el exceso en la mortalidad general, según Semanas Epidemiológicas (SE), con el objetivo de identificar cambios potencialmente asociados a la epidemia en Manaus. Los datos de mortalidad general y grupos de causas se obtuvieron en la Central de Información del Registro Civil $\mathrm{Na}$ cional y en el Sistema de Información sobre Mortalidad, referentes a 2018, 2019 y 2020. Se analizó franja de edad, sexo, lugar donde se produjo el fallecimiento, SE, año-calendario y causas de muerte. Se calcularon las causas entre las muertes acaecidas en 2019/2018 y 2020/2019 para evaluar el exceso de muertes, con intervalos de confianza en el nivel de 5\%. No se observó un exceso de mortalidad general significativo en las causas 2019/2018, independientemente de la SE. Ya las causas de 2020/2019 pasaron de 1,0 (IC95\%: 0,9-1,3) en la SE 12 a 4,6 (IC95\%: 3,9-5,3) en la SE 17. Se observó un exceso de mortalidad general con la progresión de la edad, especialmente en individuos con 60 años y más, quienes concentraron un 69,1\% (IC95\%: 66,8-71,4) de las muertes. La razón 2020/2019 para óbitos en domicilio/vía pública fue de 1,1 (IC95\%: 0,7-1,8) en la SE $12 y$ de 7,8 (IC95\%: 5,4-11,2) en la SE 17. La explosión de la mortalidad general en Manaus y la elevada proporción de óbitos en domicilio/vía pública expone la gravedad de la epidemia en contextos de gran desigualdad social y débil efectividad de las acciones gubernamentales, en especial aquellas dirigidas al combate de las desigualdades sociales y para la garantía y fortalecimiento del Sistema Único de Salud.

COVID-19; Poblaciones Vulnerables; Vigilancia en Salud Pública; Mortalidad
Recebido em 12/Mai/2020

Versão final reapresentada em 21/Mai/2020 Aprovado em 23/Mai/2020 